\title{
Antenatal management of congenital diaphragmatic hernia today and tomorrow
}

Lennart Van der Veeken ${ }^{1,2}$, Francesca Russo ${ }^{1,2}$, David Basurto ${ }^{1,2}$, Dyuti Sharma ${ }^{1}$, Tram Nguyen ${ }^{1}$, Johannes Van der Merwe ${ }^{1,2}$, Patrice Eastwood ${ }^{1,3,5}$, Namesh Khoshgoo ${ }^{3}$, Jaan Toelen ${ }^{1}$, Karel Allegaert ${ }^{1}$, Philip DeKoninck ${ }^{4,}{ }^{*}$, Stuart Hooper ${ }^{4}$, Richard Keijzer ${ }^{3}$, Paolo De Coppi $^{5,6}$, Jan Deprest ${ }^{1,2,6}$

${ }^{1}$ Academic Department of Development and Regeneration, Cluster Woman and Child, Biomedical Sciences

${ }^{2}$ Clinical Department of Obstetrics \& Gynaecology, KU Leuven, Leuven, Belgium

${ }^{3}$ Department of Surgery, Division of Pediatric Surgery, University of Manitoba, Winnipeg, Manitoba, Canada

${ }^{4}$ The Ritchie Centre, Hudson Institute of Medical Research and Department of Obstetrics and Gynaecology, Monash University, Melbourne, Victoria, Australia

${ }^{5}$ Institutes for Child Health, University College London, London, UK

${ }^{6}$ Institute for Women's Health, University College London, London, UK

This is the summary of a lecture at the International Workshop on New Trends in Pediatric Surgery, Messina (Italy), October $25^{\text {th }}, 2017$

\section{Correspondence}

Jan Deprest, MD, PhD, FRCOG: Herestraat 49, 3000 Leuven, Belgium; jan.deprest@uzleuven.be

\section{Acknowledgments}

We thank our clinical co-workers R. Devlieger, L. De Catte \& L. Lewi (UZ Leuven), and G. Attilakos \& A. David (UCLH) for their help in the clinical program. TOTAL co-investigators are thanked for their endurance in this difficult clinical trial, which has already included more than 180 patients.

\section{Conflict of interest statement}

The authors declare no conflict of interest. The KU Leuven has deposited a request for orphan designation for sildenafil to the EMA.

\section{Funding sources}

J.D.P. was a Clinical Researcher of the Flanders Research Foundation (FWO Vlaanderen; 1.8 .01207 ) and is partly funded by GOSH Children's Charity. RK is Thorlakson Chair in Surgical Research and the recipient of a Career Development Award from the Canadian Child Health Clinician Scientist Program and a New Investigator Salary Award from the Canadian Institutes of Health Research/Manitoba Lung 
Association/Children's Hospital Research Institute of Manitoba. P.D.C. is an NIHR Professor and supported by the Catapult Cell Therapy, the GOSH Children's Charity, and GOSH NIHR Biomedical Research Centre's funding scheme. Our research into CDH is funded by the KU Leuven (IOF/C3), GOSHCC, Sparks, CDHUK, Canadian Institutes of Health Research, Fetal Health Foundation (Brianna Marie) and by an Innovative Engineering for Health award by the Wellcome Trust (WT101957), the Engineering and Physical Sciences Research Council (NS/A000027/1). L.v.V., F.R., T.N., P.E. and D.B. are supported by the Erasmus+ Program of the European Commission (2013-0040). This publication reflects the views only of the author, and the Commission cannot be held responsible for any use which may be made of the information contained therein. * PDK is currently working at the Erasmus Medical Centre, Rotterdam, the Netherlands. 


\section{Introduction}

Congenital diaphragmatic hernia is a rare condition with a prevalence ranging between 1 and 4 per 10.000 pregnancies, resulting in approximately 2100 babies born each year in the EU-27 [1]. The majority of defects are left sided (85\%), a small group is right sided (13\%) and bilateral and complete agenesis are very rare (2\%). The defect arises in the embryonic period, so that abdominal organs herniate into the thorax during most of gestation, interfering with normal lung development. This leads to hypoplasia of both lungs. They have fewer and less mature airway branches and abnormal pulmonary vessels with smaller cross-sectional area, structural remodeling and altered vasoreactivity. At birth this leads to respiratory insufficiency and pulmonary hypertension (PHT). Once sufficiently stabilized the neonate undergoes surgery. Despite optimal neonatal care in specialized tertiary centers mortality rates of up to $30 \%$ remain common [2]. Survivors often suffer from medium to long term morbidities, which are poorly documented [3].

\section{Prenatal assessment}

Prenatal ultrasound screening leads to the detection of about two out of three $\mathrm{CDH}$ cases [4]. Following the diagnosis patients should be promptly referred to a tertiary center experienced in assessing and managing this anomaly in the perinatal period. It is not uncommon to find some discordance with the initial assessment in the referral center which may result in a changed perspective and different parental decisions [6]. Therefore it is considered prudent to limit prognostic advice at first diagnosis. A complete workup including advanced imaging, advanced genetic testing (we do micro-arrays) are performed to rule out associated anomalies and to guide multidisciplinary counseling to optimally inform parents for a well-funded decision about prenatal options [5]. Accurate prediction is crucial because of prenatal intervention may be contemplated. Personalized prediction is made based on the isolated nature of the defect, its side, estimation of the lung size, presence of liver herniation, and more recently stomach position may be included as well [6-12].

The lung size, used as proxy for the degree of pulmonary hypoplasia, is typically measured by 2dimensional ultrasound. It goes back on the initial proposal of Metkus et al. to measure the contralateral lung at the level of the 4-chamber view [6] and proportionate it to the head circumference measured in the standard biparietal view (lung-to-head ratio; LHR). The most accurate method for lung measurement is by tracing the lung contours. Because the lung grows faster than the head during gestation, it is inaccurate to solely rely on the absolute LHR value. Correction for gestational age is by expressing the LHR of the index case as a function of what is expected in a gestational age matched control (observed/expected LHR or o/e LHR). The formulas for calculating the o/e LHR are embedded in a freely available web-based calculator (www.totaltrial.eu) [13]. The o/e LHR has been validated for its prognostic value in 354 fetuses with unilateral isolated CDH assessed between 18 and 38 weeks gestation [14]. Its predictive value in terms of morbidity was evaluated as well $[10,15]$. Most prenatal management centers use a combination of the presence of liver herniation and the o/e LHR to divide fetuses into different groups with increasing degree of pulmonary hypoplasia and corresponding mortality. In left sided CDH (LCDH) an o/e LHR $<25 \%$ is defined as severe, and the next severity group is misleadingly called "moderate" as survival is limited to $50-60 \%$ with significant morbidity (Figure 1). In right sided CDH (RCDH), severe hypoplasia is defined as an o/e LHR $<45 \%$ (Figure 1). Besides survival, lung size and liver herniation can also predict neonatal morbidity in terms of assisted ventilation, need for supplemental oxygen, need for patch repair and time to full enteral feeding $[10,16]$. These factors are in some studies also indicative of PHT [16, 17]. Obviously magnetic resonance imaging (MRI) can be used to measure lung size as well. MRI is less influenced by maternal 
habitus, amniotic fluid quantity and allows for accurate measurement of both lungs. Volumetry can also be used to quantify liver and stomach herniation. Despite these advantages until today it has not yet been proven that MRI better predicts outcome. We recently performed a meta-analysis on the ability of (o/e) LHR, lung volume and liver herniation to predict outcome, including ECMO use and occurrence of persistent PHT [18]. The latter could not be predicted, yet both o/e LHR as well as lung volume, next to liver position, were indicative of ECMO (which on itself is a controversial treatment modality). Therefore nowadays ultrasound remains the modality on which decisions regarding prenatal management are based within clinical trials.

\section{Prenatal Intervention}

The current clinical strategy to trigger lung growth is by percutaneous fetoscopic endoluminal tracheal occlusion (FETO) (summarized in $[19,20])$. Clinical observations that fetuses with laryngeal atresia have larger lungs led to animal experiments demonstrating that fetal tracheal occlusion (TO) reverses experimental pulmonary hypoplasia [21]. TO prevents egress of lung fluid, thereby increasing airway pressure activating stretch receptors and inducing cellular proliferation. In experimental conditions sustained TO markedly reduced the number of type II pneumocytes hence surfactant expression. This can be prevented by in utero release of the TO ("plug-unplug sequence") [22]. This strategy was clinically translated, and invasiveness was markedly reduced by making this a percutaneous endoscopic procedure, which can be done under local anesthesia [23] [24]. Appropriate instruments were developed with support of the European Commission and the technique was standardized for use in a clinical trial [25].

\section{Balloon insertion or plug}

FETO is performed under sono-endoscopic guidance. The patient is positioned dorsal supine with lateral tilt to prevent caval compression. External manipulation of the fetus may be required to achieve direct access to the fetal mouth. A combination of fentanyl, curare and atropine are administered to the fetus for analgesia, immobilization and the prevention of bradycardia. A thin-walled flexible canula is placed in an area devoid of placenta with the tip targeting to or above the fetal nose (Figure 2). The fetoscope (1.3mm, Karl Storz) housed within a curved $3.3 \mathrm{~mm}$ sheath (Karl Storz) is advanced into the trachea until the carina is visualized. A detachable balloon with integrated one-way valve (Goldbal2, Balt, Montmorency, France) is placed between carina and vocal cord using a delivery catheter (BaltacciBDPE-100 0.9mm; Balt) and filling volume of $0.6 \mathrm{~mL}$. This typically only takes 10 minutes (range: 3-93 $\mathrm{min}$ ), depending on operator experience. Experience is directly related to the risk of chorionic membrane separation and amniorrhexis. The balloon appears on ultrasound as a hypoechoic fluid filled structure without colour Doppler flow. Within one week following FETO the fetal lung in responders becomes hyperechogenic [26].

\section{Balloon removal or unplug}

Reversal of occlusion is planned at 34 weeks. Leaving tracheal occlusion until delivery may provide additional lung growth yet does not increase the risk for preterm delivery from the second intervention as recently demonstrated $[27,28]$. Prenatal removal triggers lung maturation, hence lowers neonatal morbidity and improves survival $[10,28,29]$. It makes vaginal birth possible, but another important argument is that it enables birth at the initially recruiting tertiary center. Delaying removal may lead to more emergency removals at the time of birth, which are challenging and risky. Unplugging can be achieved by fetoscopy, by ultrasound-guided puncture, tracheoscopic removal on placental circulation (Figure 3) or by postnatal puncture. Fetoscopic balloon removal happens in essence in the same way 
as balloon insertion. The balloon is punctured with a stylet and can be removed using the forceps. Fetoscopy obviously confirms an unobstructed airway. Ultrasound guided puncture is also done with fetal immobilization and fetal analgesia. Because of the increased pressure and fluid entrapment, the punctured balloon will be expulsed to the mouth or amniotic cavity. Tracheal patency can be confirmed by a change in tracheal diameters and flushing under ultrasound power Doppler examination.

In $28 \%$ of cases balloon removal will be indicated earlier because of impending delivery. Even then we attempt to remove the balloon in utero. If it is not possible or safe the balloon can be removed at birth on placental circulation. This is done by performing a laryngo-tracheoscopy during a modified caesarean section with the fetal head and shoulders delivered and the head in extension. The last and least preferred method is that of postnatal retrieval. This can be performed by laryngo-trachescopy, by ultrasound puncture or by blind puncture above the manubrium sterni.

The preference for one of this techniques is mainly operator dependent. In a recent report on outcomes in 302 balloon removals at three FETO-centers $67 \%$ of balloon removals were done by fetoscopy, $21 \%$ by puncture and $10 \%$ by tracheoscopy on placental circulation, and $1 \%$ ex utero . There was no difference in gestational age at delivery whether the balloon was punctured under ultrasound guidance or removed by fetoscopy. Postnatal balloon removal should not be underestimated. The only neonatal deaths directly due to balloon removal difficulties happened when delivery took place in an unprepared and/or inexperienced environment [28].

\section{Outcomes of FETO}

The initial target group were fetuses with the worst outcome (Figure 1). Outcomes of over 200 interventions have been reported by our group. Compared to historical data from the antenatal CDH registry, FETO increased survival in severe LCDH (o/e LHR <25\%) from $24 \%$ to $49 \%$ [14]. In a recent update on RCDH, survival went from $17 \%$ to $42 \%$ in case of o/e LHR $<45 \%$ [12]. FETO also seems to improve neonatal respiratory morbidity. The strongest predictors of survival are o/e LHR before the procedure and gestational age at delivery. The latter is mainly decreased by the increased risk for preterm birth and PPROM, which occurred in $17 \%$ of cases within 3 weeks of FETO. Median delivery occurred at 35.3 weeks and $30 \%$ delivered before 34 weeks. Interestingly survival in the group delivering at 32 to 34 weeks was equal to the group delivering after 34 weeks [29]. Survivors have lower short-term morbidity which lies closer to that of moderate cases that were managed expectantly $[10,30]$.

Reported adverse events and side effects from FETO are rare. Fetal tracheomegaly is common but this does not seem to have a clinical impact except for a barking couch which fades over time [31-33]. There are only a few neonates who have had significant long term tracheal issues and these seem to be related to very early balloon insertion $<26$ weeks or to traumatic balloon removal.

Though these initial results, trials conducted by others [34] as well as a meta-analysis show effect of fetal therapy [35], leading European, U.S., Australian, and Japanese centers offer FETO only within the framework of a randomized controlled trial (RCT) TOTAL-trial (Tracheal Occlusion to Accelerate Lung Growth; www.totaltrial.eu) [36]. Severe cases are randomized to either expectant management during pregnancy or FETO (NCT01240057). Balloon insertion is performed at 27-30 weeks and removal is at 34 weeks. Postnatal management is standardized [37]. Balloon removal is set at 34 weeks because it is associated with higher survival [29]. Moreover survival does not increase further after 34 weeks and it prevents unexpected emergency balloon retrieval. The time point of insertion was slightly moved to term, to decrease the risk for delivery $<32$ weeks, which has a proven negative impact on survival [29]. Later insertion is at the expense of lung growth, so the balance will have to become clear after the trial $[27,38]$. There is also a trial on moderate pulmonary hypoplasia in LCDH (NTC00763737) comparing if 
FETO lowers oxygen need at 6 months of age. In this group, occlusion is done at 30 to 32 weeks and removal is kept at 34 weeks. This trial has been particularly successful, with currently already $85 \%$ of the sample size recruited. The "severe" TOTAL trial started later, because of ethical issues $[39,40]$.

\section{Ongoing research}

Many researchers are working on more potent and less invasive therapies for different reasons. First, the maximum post-FETO survival reported in severe cases is $50-60 \%$, so a more potent strategy would be welcomed. This is in part due to insufficient airway growth and, above all, limited improvement of vascular development. PHT has become the major cause of mortality and morbidity in $\mathrm{CDH}$. The presence and severity of PHT predicts pulmonary morbidity and death [41, 42]. PHT is also an economical burden, because costs in newborns with PHT are higher, mainly due to increase in use of extracorporeal membrane oxygenation, cost of medication and increased risk for readmission [43]. Another concern is that FETO is logistically complex, hence not universally applicable. Therefore preferentially new treatments should be medical rather than surgical.

Many researchers are working on novel medical strategies, and some are close to clinical application. Whether they will be stand-alone therapies or to be used in conjunction with fetal surgery will have to be demonstrated. Below we summarize what we are working on, yet acknowledge efforts by others.

\section{Sildenafil}

Sildenafil is a selective inhibitor of phosphodiesterase-5 (PDE5), which specifically degrades cyclic guanosine monophosphate (cGMP). PDE5 is abundantly secreted during fetal life where it acts as a key regulator of the perinatal pulmonary circulation. Sildenafil causes vasodilation, yet also promotes pulmonary angiogenesis and inhibits pulmonary artery remodeling[44] (Figure 4). It is effective and well tolerated in children affected by PHT $[45,46]$ as well as women with PHT. It has also been given to women with fetal growth restriction, pre-eclampsia and idiopathic oligohydramnios [47-50], demonstrating its safety. The rationale therefore is that sustained prenatal maternal sildenafil administration may prevent vascular remodeling associated with $\mathrm{CDH}$. This was initially demonstrated in nitrofen rats by Thebaud et al [51] and we confirmed that in a higher species. In a placebo-controlled trial transplacental sildenafil was given to fetal rabbits with and without CDH. Subcutaneous administration of sildenafil during the last three stages of lung development was safe and effective $[52,53]$. At birth, sildenafil exposed $\mathrm{CDH}$-pups had a normal vascular wall thickness, peripheral muscularization and a normal number of vessels of the fifth order or higher (thus distal). Functionally, the lungs were more compliant and had normal Doppler flow studies. All this suggest that sildenafil is a reasonable option for clinical trials in $\mathrm{CDH}$ cases. In view of this, we applied to (and received from) the European Medical Agency for orphan designation of this drug for the prevention of PHT in CDH. We meanwhile demonstrated in an ex-vivo placenta perfusion model that sildenafil crosses the placenta independent from initial maternal concentration [54].

\section{Future other possible antenatal solutions}

Stem-cell-based strategies could also ameliorate lung development and have an impact on both fetal and neonatal survival $[55,56]$. Mesenchymal stem cells (MSCs), can be derived from various tissues and are able to modulate organ repair through a paracrine pathway [57]. MSCs may even promote regeneration by differentiation into specialized cells within the lung [58]. Amniotic fluid stem (AFS) cells are broadly multipotent stem cells $[59,60]$ which have shown potential benefit in lung regeneration.[61] [62]. Rat amniotic fluid stem (AFS) cells did integrate in injured lungs and influence recovery in nitrofen-exposed rats. Both lung explants transplanted with AFS cells, or in utero transplantation of AFS cells promote lung recovery through a paracrine action, probably by the 
secretion of factors such as FGF-10, VEGF $\alpha$, and TGF- $\beta 1$. [63] Interestingly, nitrofen explants exposed to amniotic fluid cells showed significant up-regulation of surfactant protein $\mathrm{C}$ to levels observed in normal fetal lungs [64]. This was also confirmed in the more clinical relevant yet surgical rabbit model, when combined with tracheal occlusion (TO). Xenogeneic derived cells (human AFSC) reversed the lung-to-body weight ratio (LBWR), mean terminal bronchiole density (MTBD; inversely related to the number of alveoli) and medial thickness to normal without any signs of toxicity [65] [66]. This positive effect was also observed when lung derived rat MSCs were tested in the nitrofen-induced rat model. Specifically, the alveolar and the media of the pulmonary arteries were significantly thinner in the MSCtreated $\mathrm{CDH}$ rat pups than in the $\mathrm{CDH}$ fetuses not exposed to cells, therefore reducing the severity of pulmonary hypoplasia. [67]

Other tools developed within regenerative medicine may also play a relevant clinical role in the future. They are essentially based on delivering through smart synthetic materials such as nano-particles or gels growth factors or genes which are deficient in $\mathrm{CDH}$. Recently, we compared the fetal in vivo microRNA signature in hypoplastic lungs of human fetuses with severe isolated congenital diaphragmatic hernia (CDH) to changes in tracheal and amniotic fluid of fetuses undergoing FETO to reverse severe lung hypoplasia due to $\mathrm{CDH}$. Interestingly, $\mathrm{CDH}$ lungs display an increased expression of miR-200b and miR-10a as compared to control lungs. Fetuses undergoing FETO display increased miR-200 expression in their tracheal fluid at the time of balloon removal. Moreover, it was observed that future survivors of FETO display significantly higher miR-200 expression than those with a limited response lung hypoplasia and condition respiratory function through TGF-beta/SMAD signaling, the ultimate target of miR-200. [68] This concept was further investigated in the nitrofen rat. We evaluated miR-200b as a lung growth promoting therapy ex vivo and in vivo using lung explant culture and transplacental prenatal therapy in the nitrofen rat model. We could demonstrate that late lung hypoplasia in CDH was associated with (compensatory) upregulation of miR-200b in less hypoplastic lungs. Increasing miR-200b abundance with mimics early after nitrofen exposure decreases SMADdriven TGF- $\beta$ signaling and rescues lung hypoplasia both in vitro and in vivo. Also, prenatal miR-200b therapy decreases the observed incidence of $\mathrm{CDH}$. In summary, miR-200b improves pulmonary hypoplasia and decreases the incidence of $\mathrm{CDH}$ in this model [69]. This will now been taken further to higher species.

One other area which holds promises for the future of the most difficult $\mathrm{CDH}$ patients is whole lung tissue engineering. Different to simpler organs such as the bladder[70] and trachea[71], it will require more preclinical work before benefiting patients, however it has rapidly advanced beyond proof of concept studies in rodents and now preliminary successful large animal studies have been reported.[72] Particularly attractive is the possibility of using decellularised matrices and direct induced pluripotent stem cells and lung progenitor cells. This could indeed be the ultimate alternative to lung transplantation. [73] Interestingly, xenogenic decellularised matrices with patient-derived cells have lately been considered as an option for transplantation because decellularised matrices failed to generate a xenogeneic response [74]. While human lungs rejected from transplantation could in fact provide the optimal biologic scaffolds for lung tissue engineering, their availability remains very limited. Porcine lungs have been shown to become decellularised effectively using 3-[(3Cholamidopropyl)dimethylammonio]-1-propanesulfonate (CHAPS). They supported the culture of pulmonary cells in a similar way to human derived decellurised scaffolds. [75] Even very recently this approach led to the engineering and transplantation of viable lung grafts based on decellularised porcine lung scaffolds and human endothelial and epithelial cells. [76] However, for lung engineering 
species dependent cell-matrix interactions seems to exists. In a recent study, decellularised scaffolds derived from the rat, pig, primate and human lungs were shown to be different in term of stiffness, elastin and glycosaminoglycans contents. As a consequence, the adhesion of human endothelial cells was markedly enhanced on human and primate tissues when compared to decellularised lungs derived from other species which has important implications on attempts at lung regeneration. [77]

When considering the candidate cellular component, both multipotent or pluripotent cell have been adopted. In humans, proliferative KRT5(+)TP63(+) basal epithelial stem cell can be isolated and used to repopulate both acellular rat and human scaffolds in ex vivo whole organ culture. After seeding, the cells continue to proliferate and, in combination with primary pulmonary endothelial cells, functionally differentiate displaying dynamic compliance and rudimentary gas exchange capacity. [78] Alternatively, alveolar epithelial cells (AECs) can be differentiated from induced pluripotent stem cells (iPSCS). Alveolar epithelial type II (AETII) and type I (AETI) cells generated from human iPSCs have phenotypic properties similar to those of mature human AETII and AETI cells. When seeded on acellular rat or human lung matrices, these progenitor cells adhered to and proliferated within the 3D lung tissue scaffold and displayed markers of differentiated pulmonary epithelium. [79] Similarly, in engineered lungs using iPS derived cells, up to $97 \%$ of cells were positive for surfactant protein C, $95 \%$ for mucin-1, 93\% for surfactant protein B, and $89 \%$ for the epithelial marker CD54. Under appropriate culture conditions, these progenitor cells not only adhered and proliferated within the 3D lung tissue scaffold, but also responded to physiological stimuli such as change of phenotype from AET type II to type I when exposed to a Wnt/ $\beta$-catenin inhibitor (IWR-1). Moreover, when seeded and cultured in a decellularised mouse lung scaffold, they reformed an alveolar structure and expressed surfactant protein C (SPC) or T1 $\alpha$ protein (markers of AET II or AET I cells, respectively). [80] Similar approaches have been used also to regenerate a functional pulmonary vasculature by repopulating the vascular compartment of decellularised rat and human lung scaffolds with human cells, including endothelial and perivascular cells derived from induced pluripotent stem cells. The regenerated endothelium not only showed reduced vascular resistance and improved barrier function over the course of in vitro culture, but remained patent for 3 days after orthotopic transplantation in rats. [81]

\section{Conclusion}

In the era of prenatal diagnosis of $\mathrm{CDH}$, patients should receive individualized counseling on the expected outcome. Fetoscopic endoluminal tracheal occlusion is clinically feasible and may improve outcomes. We consider it as an investigational procedure that may alter the natural course of the disease. This hypothesis is tested in ongoing TOTAL-trial. As FETO remains an invasive technique, it is associated with a significantly increased risk for preterm birth which potentially tempers its benefits. Our current research projects stretch further than the surgical intervention. Medical interventions may be the first one to move into clinical practice, yet other strategies are in the translational pipeline as well. 
Figure 1: Stratification of fetuses with isolated CDH based on o/e LHR and for LCDH (A) presence or absence of liver herniation. In RCDH (B) liver herniation is (nearly) always present. The yellow arrows indicate to what level survival increases based on some case series.

Figure 2: Fetal Endoscopic Tracheal Occlusion (FETO): schematic drawing showing, access to uterus and the fetal trachea. Inserts: steps in balloon delivery. Reproduced with permission from UZ Leuven, Leuven, Belgium. Drawing: Myrthe Boymans.

Figure 3: Schematic drawing of balloon removal on placental circulation by laryngeo-tracheoscopy. Reproduced with permission from UZ Leuven, Leuven, Belgium. Drawing: Myrthe Boymans.

Figure 4: Schematic representation of the mechanism of action of sildenafil on the pulmonary vasculature. eNOS: endothelial nitric oxide synthase; NO: nitric oxide; GC: guanylate cyclase; GTP: guanosine triphosphate; GMP: guanosine triphosphate; cGMP: cyclic GMP; PDE: phosphodiesterase; ATP: adenosine triphosphate; AMP: adenosine monophosphate; cAMP: cyclic AMP; PKG: cGMP dependent protein kinase; PKA: cAMP dependent protein kinase. Reproduced with permission from UZ Leuven, Leuven, Belgium. Drawing Myrthe Boymans. 
Table 1: Fetal criteria for isolated left sided CDH and list of outcome measurements in the TOTAL trial

\begin{tabular}{|c|c|}
\hline Trial for severe hypoplasia & Trial for moderalte hypoplasia \\
\hline & \\
\hline \multicolumn{2}{|c|}{$\begin{array}{l}\text { Primary outcome measurement } \\
\text { - Survival (in case of severe hypoplasia) without bronchopulmonary dysplasia [82] (in case of moderate hypoplasia) } \\
\text { Secondary postnatal outcome measurements } \\
\text { - Survival at discharge from the hospital } \\
\text { - Grade of oxygen dependency (grades 0-III) } \\
\text { - Occurrence of severe pulmonary hypertension } \\
\text { - Need for ECMO support for centers offering it } \\
\text { - Number of days in neonatal intensive care unit } \\
\text { - Number of days of ventilatory support } \\
\text { - Presence of periventricular leukomalacia at } \leq 2 \text { months postnatally } \\
\text { - Presence of neonatal sepsis } \\
\text { - Presence of intraventricular hemorrhage (grades } 0 \text {-III) } \\
\text { - Presence of retinopathy of prematurity (grade } \geq 11 \text { ) } \\
\text { - Number of days till full enteral feeding } \\
\text { - Presence of gastroesophageal reflux (above one third of the esophagus on clinically indicated radiologic study) } \\
\text { - Day of surgery } \\
\text { - Use of a patch (yes/no) } \\
\text { Secondary prenatal outcome measure } \\
\text { - Lung volume as serially measured (O/E LHR, O/E total lung volume) } \\
\text { - Liver position; in case of liver herniation, the liver-to-thorax volume } \\
\text { Secondary longer-term outcome variables } \\
\text { - Pulmonary function and volume testing before and after repair, at discharge, and at } 1 \text { y of age } \\
\text { - Neurodevelopmental milestones at discharge, } 12 \text { months, and } 2 \text { y (ages and stages) } \\
\text { - Death at } 2 \text { y (caused by either primary illness or other causes) }\end{array}$} \\
\hline & \\
\hline
\end{tabular}


1. EUROCAT, EUROCAT Statistical Monitoring Report - 2012.

2. van den Hout, L., et al., Actual outcome in infants with congenital diaphragmatic hernia: the role of a standardized postnatal treatment protocol. Fetal Diagn Ther, 2011. 29(1): p. 55-63.

3. Bagolan, P. and F. Morini, Long-term follow up of infants with congenital diaphragmatic hernia. Semin Pediatr Surg, 2007. 16(2): p. 134-44.

4. Gallot, D., et al., Prenatal detection and outcome of congenital diaphragmatic hernia: a French registry-based study. Ultrasound Obstet Gynecol, 2007. 29(3): p. 276-83.

5. Deprest, J., et al., Current consequences of prenatal diagnosis of congenital diaphragmatic hernia. J Pediatr Surg, 2006. 41(2): p. 423-30.

6. Metkus, A.P., et al., Sonographic predictors of survival in fetal diaphragmatic hernia. J Pediatr Surg, 1996. 31(1): p. 148-51; discussion 151-2.

7. Kitano, Y., et al., Re-evaluation of stomach position as a simple prognostic factor in fetal left congenital diaphragmatic hernia: a multicenter survey in Japan. Ultrasound Obstet Gynecol, 2011. 37(3): p. 277-82.

8. Mayer, S., et al., The correlation between lung volume and liver herniation measurements by fetal MRI in isolated congenital diaphragmatic hernia: a systematic review and meta-analysis of observational studies. Prenat Diagn, 2011. 31(11): p. 1086-96.

9. Cannie, M., et al., Quantification of intrathoracic liver herniation by magnetic resonance imaging and prediction of postnatal survival in fetuses with congenital diaphragmatic hernia. Ultrasound Obstet Gynecol, 2008. 32(5): p. 627-32.

10. Done, E., et al., Predictors of neonatal morbidity in fetuses with severe isolated congenital diaphragmatic hernia undergoing fetoscopic tracheal occlusion. Ultrasound Obstet Gynecol, 2013.

11. Cordier, A.G., et al., Stomach position versus liver-to-thoracic volume ratio in left-sided congenital diaphragmatic hernia. J Matern Fetal Neonatal Med, 2015. 28(2): p. 190-5.

12. DeKoninck, P., et al., Right-sided congenital diaphragmatic hernia in a decade of fetal surgery. BJOG, 2015. 122(7): p. 940-6.

13. Dekoninck, P., et al., Results of fetal endoscopic tracheal occlusion for congenital diaphragmatic hernia and the set up of the randomized controlled TOTAL trial. Early Hum Dev, 2011. 87(9): p. 619-24.

14. Jani, J., et al., Observed to expected lung area to head circumference ratio in the prediction of survival in fetuses with isolated diaphragmatic hernia. Ultrasound Obstet Gynecol, 2007. 30(1): p. 67-71.

15. Jani, J.C., et al., Prenatal prediction of neonatal morbidity in survivors with congenital diaphragmatic hernia: a multicenter study. Ultrasound Obstet Gynecol, 2009. 33(1): p. 64-9.

16. Done, E., et al., Prediction of neonatal respiratory function and pulmonary hypertension in fetuses with isolated congenital diaphragmatic hernia in the fetal endoscopic tracleal occlusion era: a single-center study. Fetal Diagn Ther, 2015. 37(1): p. 24-32.

17. Lusk, L.A., et al., Fetal ultrasound markers of severity predict resolution of pulmonary hypertension in congenital diaphragmatic hernia. Am J Obstet Gynecol, 2015. 213(2): p. 216 e1-8.

18. Russo, F.M., et al., Lung size and liver herniation predict need for extracorporeal membrane oxygenation but not pulmonary hypertension in isolated congenital diaphragmatic hernia: systematic review and meta-analysis. Ultrasound Obstet Gynecol, 2017. 49(6): p. 704-713.

19. Deprest, J.A., et al., The making of fetal surgery. Prenat Diagn, 2010. 30(7): p. 653-67.

20. Khan, P.A., M. Cloutier, and B. Piedboeuf, Tracheal occlusion: a review of obstructing fetal lungs to make them grow and mature. Am J Med Genet C Semin Med Genet, 2007. 145C(2): p. 125-38.

21. Wilson, J.M., J.W. DiFiore, and C.A. Peters, Experimental fetal tracheal ligation prevents the pulmonary hypoplasia associated with fetal nephrectomy: possible application for congenital diaphragmatic hernia. J Pediatr Surg, 1993. 28(11): p. 1433-9; discussion 1439-40. 
22. Flageole, H., et al., The plug-unplug sequence: an important step to achieve type II pneumocyte maturation in the fetal lamb model. J Pediatr Surg, 1998. 33(2): p. 299-303.

23. Deprest, J., et al., Fetoscopic tracheal occlusion (FETO) for severe congenital diaphragmatic hernia: evolution of a technique and preliminary results. Ultrasound Obstet Gynecol, 2004. 24(2): p. 121-6.

24. Jani, J.C., et al., Severe diaphragmatic hernia treated by fetal endoscopic tracheal occlusion. Ultrasound Obstet Gynecol, 2009. 34(3): p. 304-10.

25. Van der Veeken L, et al. Gynecol Surg 2018 (in press)

26. Peralta, C.F., et al., Fetal lung volume after endoscopic tracheal occlusion in the prediction of postnatal outcome. Am J Obstet Gynecol, 2008. 198(1): p. 60 e1-5.

27. Nawapun, K., et al., In vivo evidence by magnetic resonance volumetry of a gestational age dependent response to tracheal occlusion for congenital diaphragmatic hernia. Prenat Diagn, 2015. 35(11): p. 1048-56.

28. Jimenez, J.A., et al., Balloon removal after fetoscopic endoluminal tracheal occlusion for congenital diaphragmatic hernia. Am J Obstet Gynecol, 2017.

29. Deprest, J., et al., Technical aspects of fetal endoscopic tracheal occlusion for congenital diaphragmatic hernia. J Pediatr Surg, 2011. 46(1): p. 22-32.

30. Verbelen, T., et al., Antireflux surgery after congenital diaphragmatic hernia repair: a plea for a tailored approach. Eur J Cardiothorac Surg, 2013.

31. Breysem, L., et al., Cross-sectional study of tracheomegaly in children after fetal tracheal occlusion for severe congenital diaphragmatic hernia. Radiology, 2010. 257(1): p. 226-32.

32. Zani, A., et al., Tracheomegaly in infants with severe congenital diaphragmatic hernia treated with fetal endoluminal tracheal occlusion. J Pediatr, 2014. 164(6): p. 1311-5.

33. Fayoux, P., et al., Neonatal tracheal changes following in utero fetoscopic balloon tracheal occlusion in severe congenital diaphragmatic hernia. J Pediatr Surg, 2010. 45(4): p. 687-92.

34. Ruano, R., et al., A randomized controlled trial of fetal endoscopic tracheal occlusion versus postnatal management of severe isolated congenital diaphragmatic hernia. Ultrasound in obstetrics \& gynecology : the official journal of the International Society of Ultrasound in Obstetrics and Gynecology, 2012. 39(1): p. 20-7.

35. Al-Maary, J., et al., Fetal Tracheal Occlusion for Severe Pulmonary Hypoplasia in Isolated Congenital Diaphragmatic Hernia: A Systematic Review and Meta-analysis of Survival. Ann Surg, 2016. 264(6): p. 929-933.

36. Deprest, J., et al., Prenatal management of the fetus with isolated congenital diaphragmatic hernia in the era of the TOTAL trial. Semin Fetal Neonatal Med, 2014. 19(6): p. 338-348.

37. Snoek, K.G., et al., Standardized Postnatal Management of Infants with Congenital Diaphragmatic Hernia in Europe: The CDH EURO Consortium Consensus - 2015 Update. Neonatology, 2016. 110(1): p. 66-74.

38. Cannie, M.M., et al., Evidence and patterns in lung response after fetal tracheal occlusion: clinical controlled study. Radiology, 2009. 252(2): p. 526-33.

39. Deprest, J., et al., The fetal patient -- ethical aspects of fetal therapy. Facts Views Vis Obgyn, 2011. 3(3): p. 221-7.

40. Rodrigues, H.C., J. Deprest, and P.P. Berg, When referring physicians and researchers disagree on equipoise: the TOTAL trial experience. Prenat Diagn, 2011. 31(6): p. 589-94.

41. Vanamo, K., et al., Long-term pulmonary sequelae in survivors of congenital diaphragmatic defects. J Pediatr Surg, 1996. 31(8): p. 1096-9; discussion 1099-100.

42. Lusk, L.A., et al., Persistence of pulmonary hypertension by echocardiography predicts shortterm outcomes in congenital diaphragmatic hernia. J Pediatr, 2015. 166(2): p. 251-6 e1.

43. Sikirica, M., et al., The economic burden of pulmonary arterial hypertension (PAH) in the US on payers and patients. BMC Health Serv Res, 2014. 14: p. 676.

44. Barnett, C.F. and R.F. Machado, Sildenafil in the treatment of pulmonary hypertension. Vasc Health Risk Manag, 2006. 2(4): p. 411-22. 
45. Barst, R.J., et al., STARTS-2: long-term survival with oral sildenafil monotherapy in treatmentnaive pediatric pulmonary arterial hypertension. Circulation, 2014. 129(19): p. 1914-23.

46. Barst, R.J., et al., A randomized, double-blind, placebo-controlled, dose-ranging study of oral sildenafil citrate in treatment-naive children with pulmonary arterial hypertension. Circulation, 2012. 125(2): p. 324-34.

47. Ganzevoort, W., et al., STRIDER: Sildenafil Therapy In Dismal prognosis Early-onset intrauterine growth Restriction--a protocol for a systematic review with individual participant data and aggregate data meta-analysis and trial sequential analysis. Syst Rev, 2014. 3: p. 23.

48. Maher, M.A., T.M. Sayyed, and N. Elkhouly, Sildenafil Citrate Therapy for Oligohydramnios: A Randomized Controlled Trial. Obstet Gynecol, 2017. 129(4): p. 615-620.

49. Samangaya, R.A., et al., A randomised, double-blinded, placebo-controlled study of the phosphodiesterase type 5 inhibitor sildenafil for the treatment of preeclampsia. Hypertens Pregnancy, 2009. 28(4): p. 369-82.

50. von Dadelszen, P., et al., Sildenafil citrate therapy for severe early-onset intrauterine growth restriction. BJOG, 2011. 118(5): p. 624-8.

51. Luong, C., et al., Antenatal sildenafil treatment attenuates pulmonary hypertension in experimental congenital diaphragmatic hernia. Circulation, 2011. 123(19): p. 2120-31.

52. Russo, F.M., et al., Transplacental sildenafil rescues lung abnormalities in the rabbit model of diaphragmatic hernia. Thorax, 2016. 71(6): p. 517-25.

53. Russo, F.M., et al., Pregnancy affects the pharmacokinetics of sildenafil and its metabolite in the rabbit. Xenobiotica, 2017: p. 1-26.

54. Russo FM, Conings $\mathrm{S}$, Annaert $\mathrm{P}$, et al. Placenta transfer of sildenafil citrate in the ex-vivo human cotyledon perfusion model. Am J Obstet Gynecol 2017;216(1 Suppl):S280.

55. Jeanty, C., S.M. Kunisaki, and T.C. MacKenzie, Novel non-surgical prenatal approaches to treating congenital diaphragmatic hernia. Semin Fetal Neonatal Med, 2014. 19(6): p. 349-56.

56. Deprest, J., et al., Medical and regenerative solutions for congenital diaphragmatic hernia: a perinatal perspective. Eur J Pediatr Surg, 2014. 24(3): p. 270-7.

57. Pozzobon, M., M. Ghionzoli, and P. De Coppi, ES, iPS, MSC, and AFS cells. Stem cells exploitation for Pediatric Surgery: current research and perspective. Pediatr Surg Int, 2010. 26(1): p. 3-10.

58. Wong, A.P., et al., Identification of a bone marrow-derived epithelial-like population capable of repopulating injured mouse airway epithelium. J Clin Invest, 2009. 119(2): p. 336-48.

59. Ditadi, A., et al., Human and murine amniotic fluid c-Kit+Lin-cells display hematopoietic activity. Blood, 2009. 113(17): p. 3953-60.

60. De Coppi, P., et al., Isolation of amniotic stem cell lines with potential for therapy. Nat Biotechnol, 2007. 25(1): p. 100-6.

61. Ramachandra, D.L., et al., In utero therapy for congenital disorders using amniotic fluid stem cells. Front Pharmacol, 2014. 5: p. 270.

62. DeKoninck, P., et al., Routine isolation and expansion late mid trimester amniotic fluid derived mesenchymal stem cells in a cohort of fetuses with congenital diaphragmatic hernia. Eur J Obstet Gynecol Reprod Biol, 2014. 178: p. 157-62.

63. Pederiva, F., et al., Amniotic fluid stem cells rescue both in vitro and in vivo growth, innervation, and motility in nitrofen-exposed hypoplastic rat lungs through paracrine effects. Cell Transplant, 2013. 22(9): p. 1683-94.

64. Di Bernardo, J., et al., Amniotic fluid derived mesenchymal stromal cells augment fetal lung growth in a nitrofen explant model. J Pediatr Surg, 2014. 49(6): p. 859-64; discussion 864-5.

65. DeKoninck, P., et al., The use of human amniotic fluid stem cells as an adjunct to promote pulmonary development in a rabbit model for congenital diaphragmatic hernia. Prenat Diagn, 2015. 35(9): p. 833-40.

66. Merrell, A.J., et al., Muscle connective tissue controls development of the diaphragm and is a source of congenital diaphragmatic hernias. Nat Genet, 2015. 47(5): p. 496-504. 
67. Yuniartha, R., et al., Therapeutic potential of mesenchymal stem cell transplantation in a nitrofen-induced congenital diaphragmatic hernia rat model. Pediatr Surg Int, 2014. 30(9): p. 907-14.

68. Pereira-Terra, P., et al., Unique Tracheal Fluid MicroRNA Signature Predicts Response to FETO in Patients With Congenital Diaphragmatic Hernia. Ann Surg, 2015. 262(6): p. 1130-40.

69. Khoshgoo, N., et al., Prenatal microRNA miR-200b Therapy Improves Nitrofen-induced Pulmonary Hypoplasia Associated With Congenital Diaphragmatic Hernia. Ann Surg, 2017.

70. Atala, A., et al., The effect of gastric augmentation on bladder function. J Urol, 1993. 149(5): p. 1099-102.

71. Elliott, M.J., et al., Stem-cell-based, tissue engineered tracheal replacement in a child: a 2year follow-up study. Lancet, 2012. 380(9846): p. 994-1000.

72. Calle, E.A., et al., Lung regeneration: steps toward clinical implementation and use. Curr Opin Anaesthesiol, 2017. 30(1): p. 23-29.

73. Hoganson, D.M., E.K. Bassett, and J.P. Vacanti, Lung tissue engineering. Front Biosci (Landmark Ed), 2014. 19: p. 1227-39.

74. Fishman, J.M., et al., Immunomodulatory effect of a decellularized skeletal muscle scaffold in a discordant xenotransplantation model. Proc Natl Acad Sci U S A, 2013. 110(35): p. 14360-5.

75. O'Neill, J.D., et al., Decellularization of human and porcine lung tissues for pulmonary tissue engineering. Ann Thorac Surg, 2013. 96(3): p. 1046-55; discussion 1055-6.

76. Zhou, H., et al., Bioengineering Human Lung Grafts on Porcine Matrix. Ann Surg, 2017.

77. Balestrini, J.L., et al., Comparative biology of decellularized lung matrix: Implications of species mismatch in regenerative medicine. Biomaterials, 2016. 102: p. 220-30.

78. Gilpin, S.E., et al., Regenerative potential of human airway stem cells in lung epithelial engineering. Biomaterials, 2016. 108: p. 111-9.

79. Ghaedi, M., et al., Human iPS cell-derived alveolar epithelium repopulates lung extracellular matrix. J Clin Invest, 2013. 123(11): p. 4950-62.

80. Zhou, Q., et al., Differentiation of mouse induced pluripotent stem cells into alveolar epithelial cells in vitro for use in vivo. Stem Cells Transl Med, 2014. 3(6): p. 675-85.

81. Gubareva, E.A., et al., Orthotopic transplantation of a tissue engineered diaphragm in rats. Biomaterials, 2016. 77: p. 320-35.

82. Bancalari, E. and N. Claure, Definitions and diagnostic criteria for bronchopulmonary dysplasia. Semin Perinatol, 2006. 30(4): p. 164-70. 
A

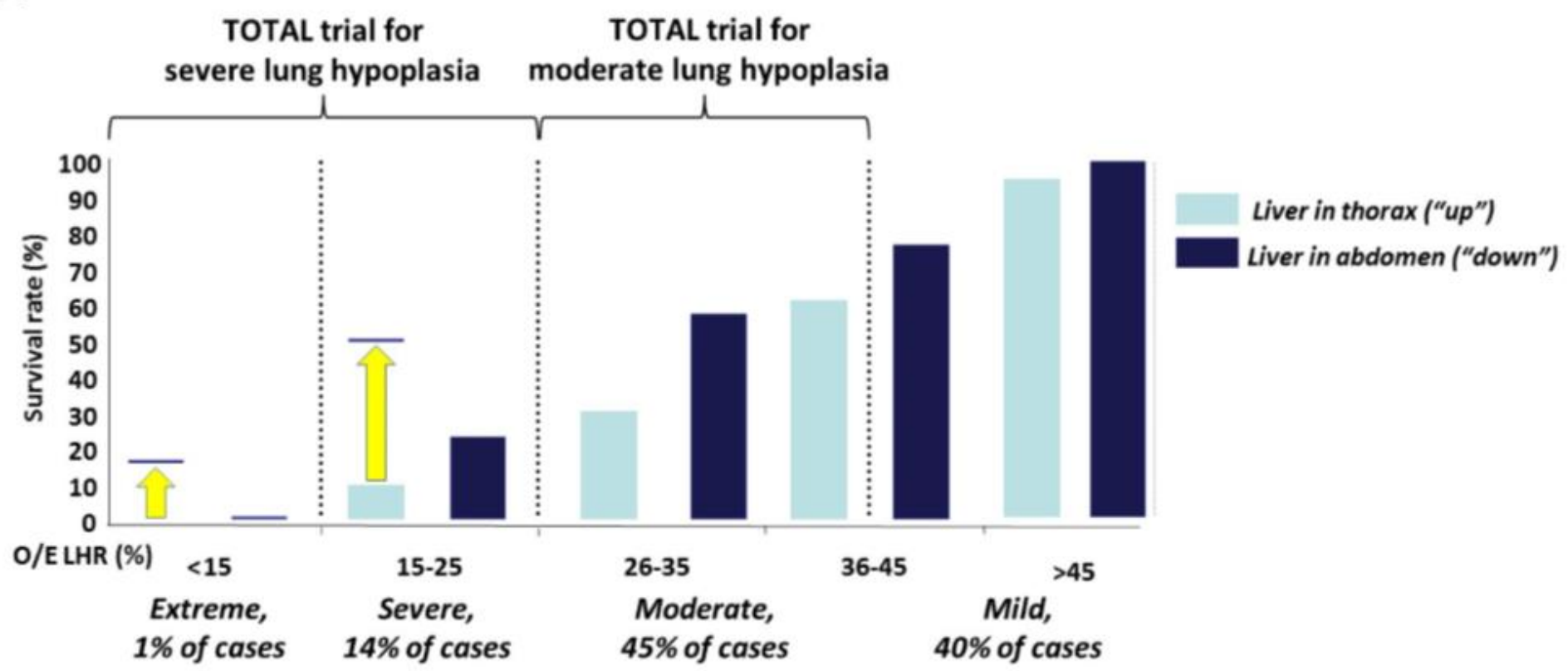

B

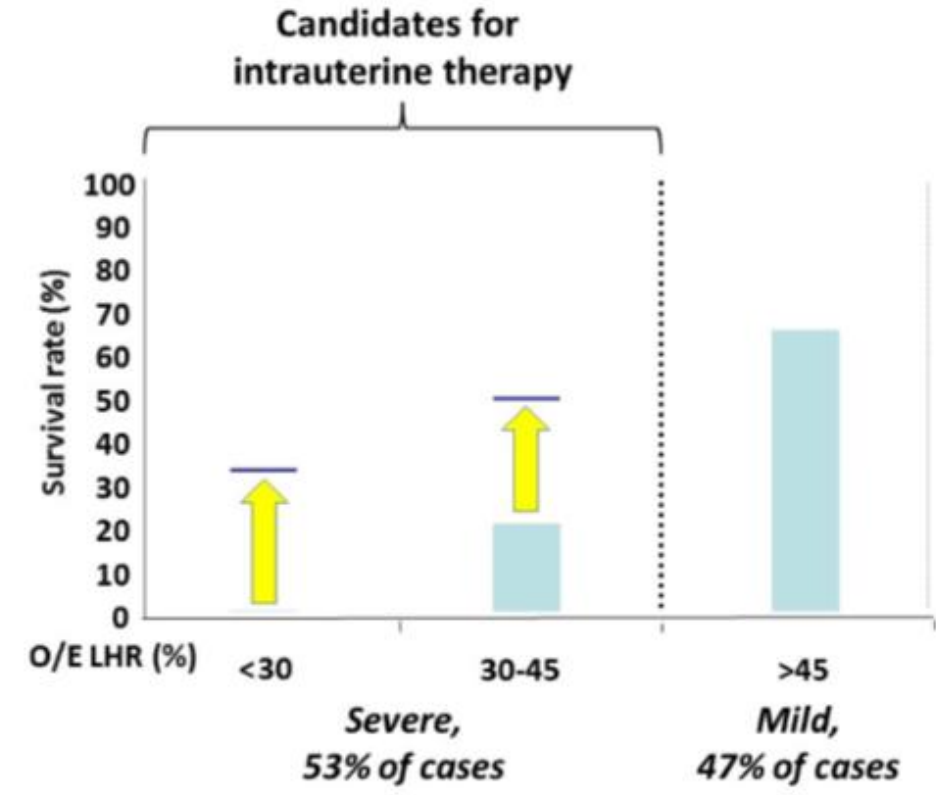

Figure 1 (available as TIFF as well) 
Figure 2

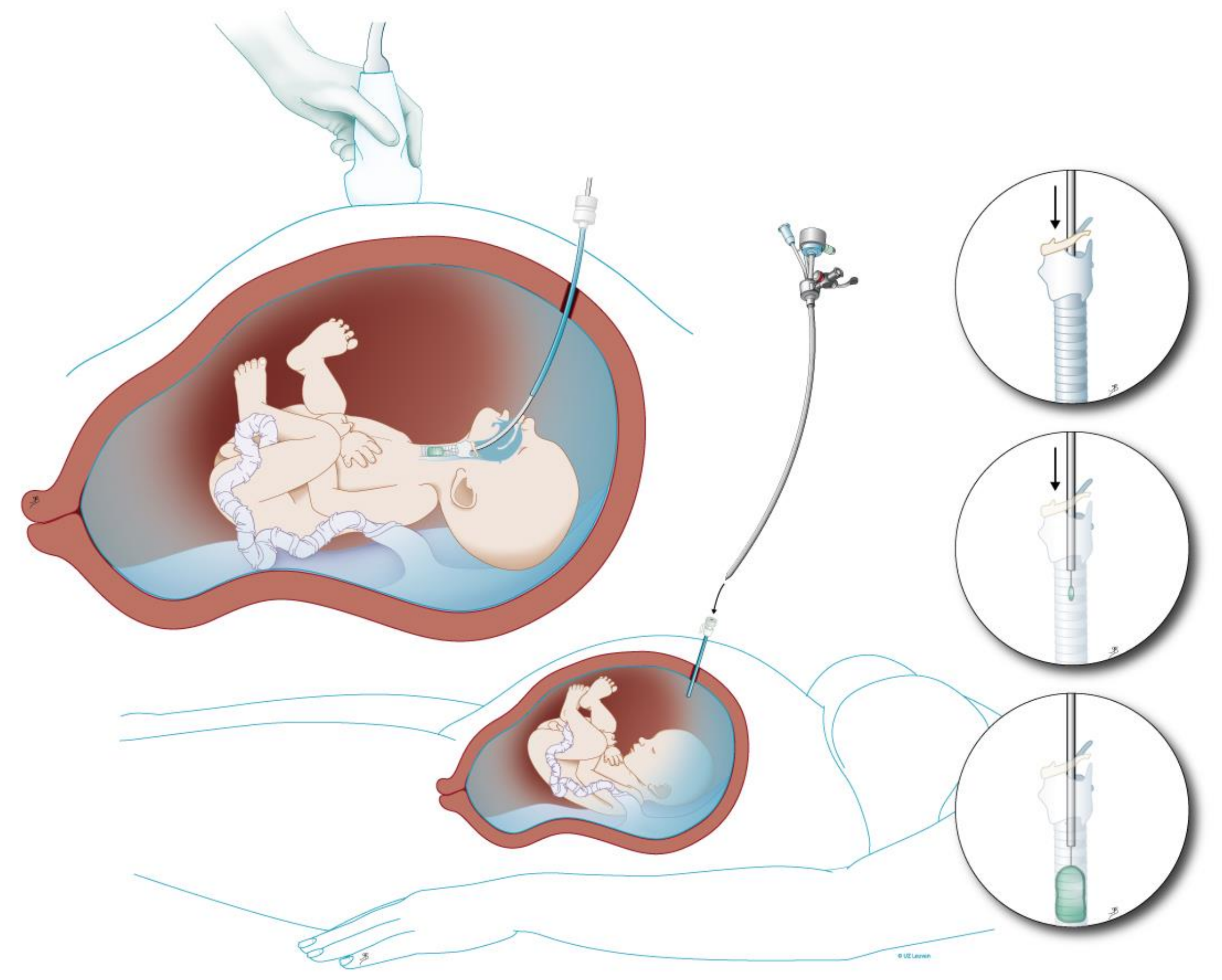


figure 3

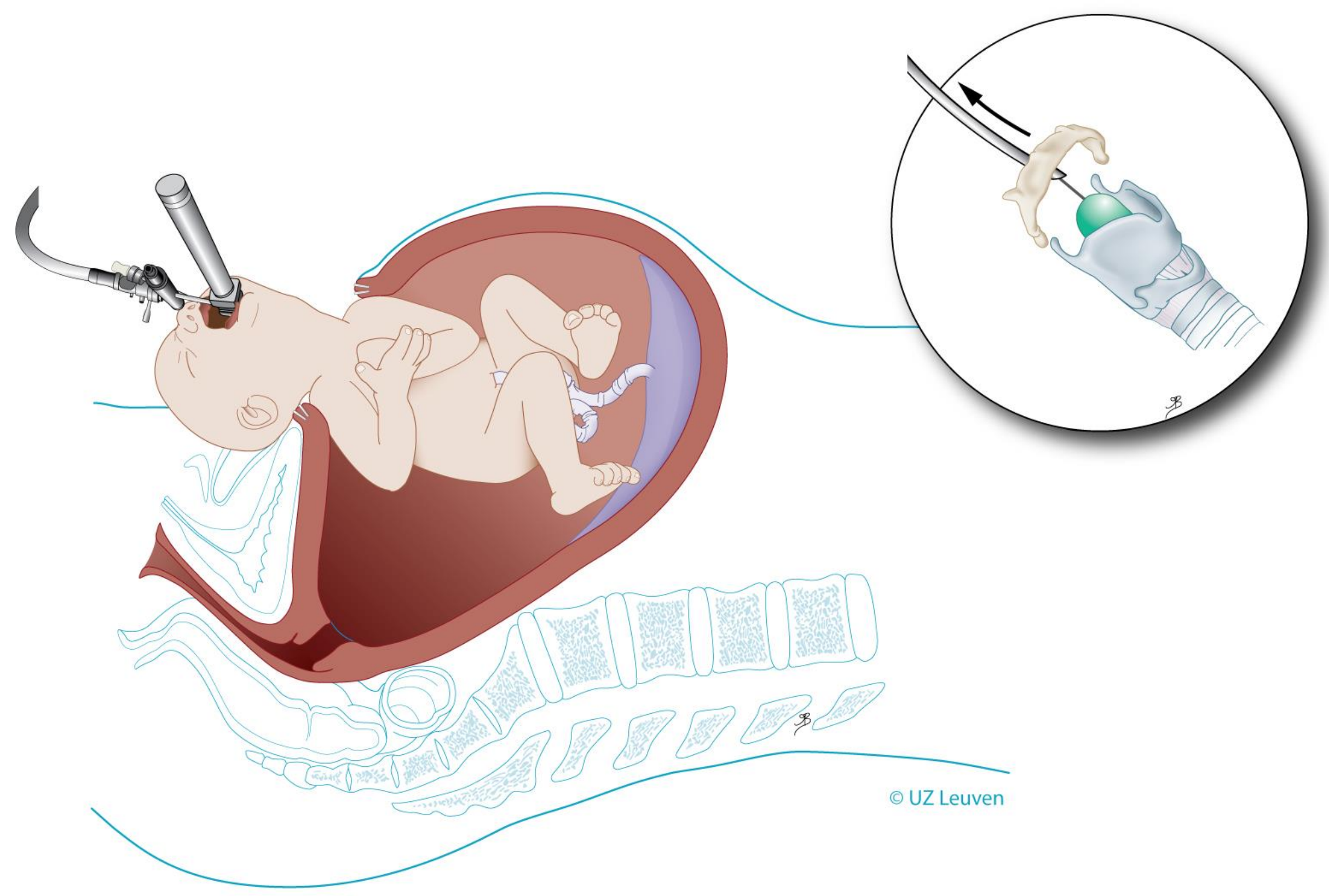



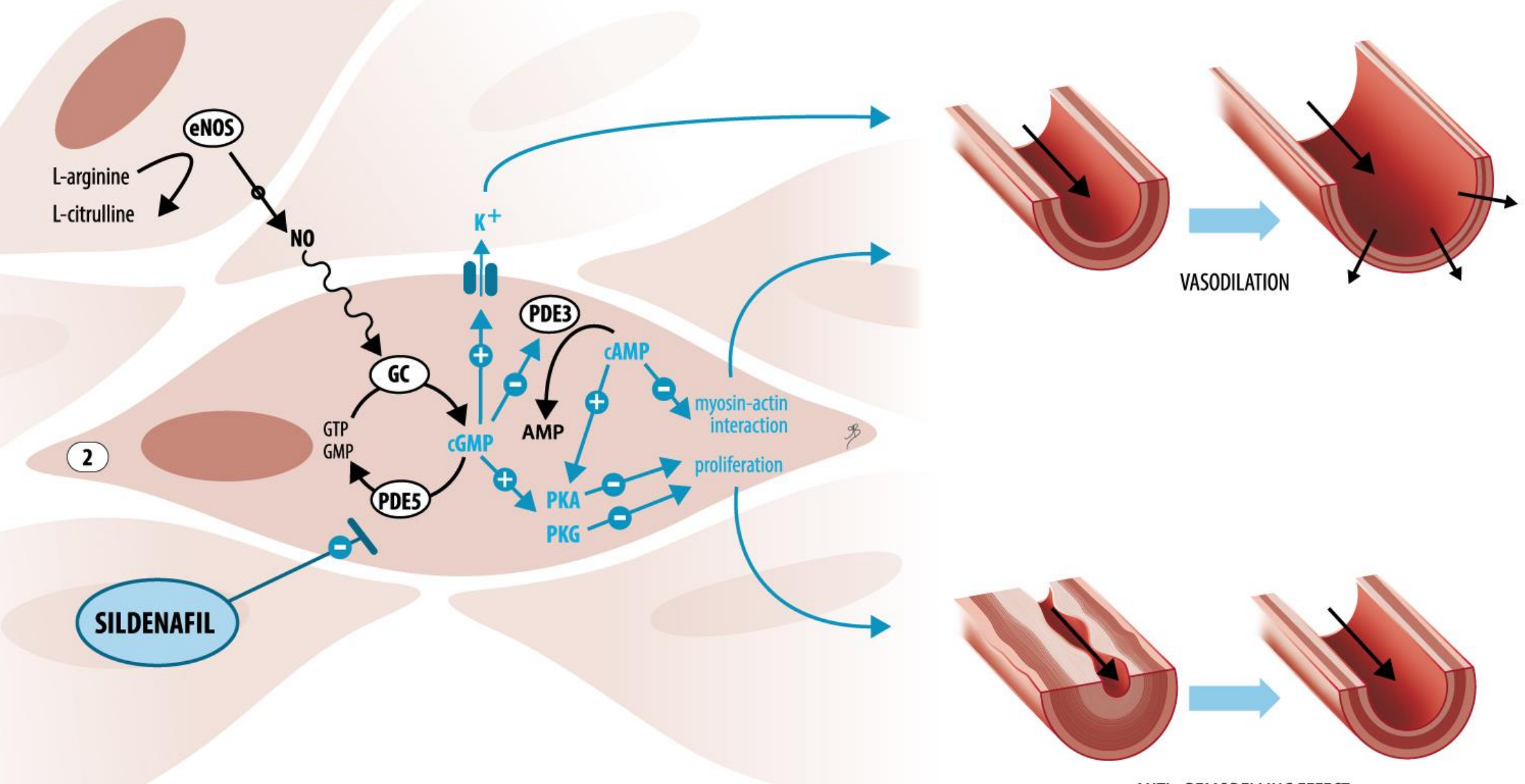

(c) UZ Leuven

ANTI- REMODELLING EFFECT 